\title{
FORUM
}

\section{Vergleichende Regionalismusforschung und Diffusion: Eine neue Forschungsagenda}

\author{
Anja Jetschke / Tobias Lenz
}

\section{Comparative Regionalism and Diffusion: A New Research Agenda}

Abstract: The number of regional organizations and regional trade agreements has risen sharply since the 1990s. In its wake, comparative research on regionalism has seen a revival. An important strand of this literature asks about the drivers of these developments, but has to date largely neglected a puzzling phenomenon: the similarities between regional organizations in their institutional design and the chosen methods of integration. Existing perspectives analyze different cases of regionalism primarily as phenomena that develop independently of each other, and whose genesis and design are determined either endogenously by domestic regional dynamics or exogenously by powerful hegemons. Against this background, this article argues for an extension of existing analytical perspectives and sketches a diffusion-oriented research agenda that conceives of regional organizations as interdependent phenomena.

Keywords: Comparative Regionalism, Diffusion, Institutional Design, EU as a Model, Regional Integration

Schlüsselwörter: Vergleichende Regionalismusforschung, Diffusion, institutionelles Design, EU als Modell, Regionalintegration

\section{Einleitung}

Seit Anfang der 1990er-Jahre hat die Zahl der Regionalorganisationen und regionalen Handelsabkommen rasant zugenommen. Im Zuge dessen hat auch die Vergleichende Regionalismusforschung neuen Aufschwung erhalten. ${ }^{1}$ Regionalismus kann hier als staatlich gelenkte Kooperation und Integration zwischen Ländern

1 Unser Dank geht an die DFG-geförderte Kollegforschergruppe „The Transformative Power of Europe" an der FU Berlin, die der Autorin und dem Autor 2009-2010 einen idealen Forschungskontext geboten hat. Unser besonderer Dank gilt Tanja Börzel und Thomas Risse und den TeilnehmerInnen des Jour Fixe. Die äußerst konstruktiven Anregungen der beiden GutachterInnen der PVS haben einzelne Argumente des Artikels erheblich verbessert. Anja Jetschke dankt außerdem dem Ministerium für Wissenschaft, Forschung und Kunst Baden-Württemberg für die Unterstützung im Rahmen des Margarete-von-Wrangell Habilitationsprogramms. Tobias Lenz bedankt sich ebenfalls bei Kalypso Nicolaïdis für viele anregende Diskussionen zum Thema dieses Artikels. 
innerhalb einer geographischen Region definiert werden. ${ }^{2}$ Ein Schwerpunkt der Literatur fragt nach den Antriebskräften dieser Entwicklung: Welche Faktoren treiben die Evolution des Regionalismus an? Wie lassen sich unterschiedliche Entwicklungsverläufe erklären? Und welche Faktoren beeinflussen ihren Erfolg oder Misserfolg? Obwohl Antworten auf und Herangehensweisen an diese Fragen zunehmend "multidimensional“ geworden sind (Sbragia 2008, S. 29), ignoriert diese Forschung bisher ein erstaunliches Phänomen: die Ähnlichkeit vieler Regionalorganisationen in ihrem institutionellen Design und den gewählten Integrationsmethoden. ${ }^{3}$ Verschiedene Autoren weisen auf solche Ähnlichkeiten in der institutionellen Struktur hin. Karen Alter und Laurence Helfer (2010) untersuchen die Praxis des Gerichtshofs der Andengemeinschaft, der eine Kopie des Europäischen Gerichtshofes (EuGH) darstellt, und erwähnen neun weitere Regionalorganisationen, die das Modell des EuGH übernommen haben. Die Gemeinschaft Südostasiatischer Staaten (ASEAN), eine der erfolgreichsten Regionalorganisationen außerhalb Europas und bisher eher als Gegenmodell zur EU bekannt, hat 2008 mit dem Komitee Ständiger Vertreter ein, dem EU-Ausschuss der Ständigen Vertreter (COREPER) ähnelndes Organ geschaffen und strebt eine wirtschaftliche Integration nach dem Vorbild der Europäischen Gemeinschaft (EG) an (Jetschke 2009). Die Organisation für Afrikanische Einheit, die 1963 als Regionalorganisation nach dem Vorbild der Vereinten Nationen gegründet wurde, beschloss 1991 einen Gemeinsamen Markt zu bilden und schuf 2000 eine Kommission und einen Gerichtshof. Die neue Afrikanische Union nähert sich der Struktur der EU in erstaunlicher Weise an (Packer u. Rukare 2002).

Diese Ähnlichkeiten zwischen Regionalorganisationen werden in der Literatur bisher nicht systematisch erfasst und theoretisiert. Bestehende Ansätze analysieren verschiedene Fälle von Regionalismus hauptsächlich als voneinander unabhängige Phänomene, die sich nicht wechselseitig beeinflussen und deren Entstehung und Design entweder endogen durch regionale Dynamiken (bottom-up) oder exogen durch mächtige Staaten bestimmt sind (top-down). Wir fassen diese beiden Perspektiven unter vertikalen Perspektiven zusammen. Vor diesem Hintergrund plädieren wir in diesem Artikel für eine Ausweitung der Analyseperspektiven in der Vergleichenden Regionalismusforschung und skizzieren eine neue Forschungsagenda, die Regionalorganisationen als interdependente Phänomene begreift. Wir bezeichnen diese Perspektive als horizontal und verstehen darunter Einflüsse im globalen Regionalismus, die sich zwischen Regionalorganisationen abspielen, mit besonderem Augenmerk auf der Rolle der Europäischen Union (EU). Wir entwickeln zwei Hauptargumente. Erstens konstatieren wir, dass die neuere Regionalismusliteratur horizontale Perspektiven bisher weitgehend vernachlässigt hat. Zweitens argumentieren wir, dass horizontale Einflüsse nützlicherweise als Diffusionsprozess im globalen Regionalismus konzeptionalisiert werden können - eine

2 Regionalismus als staatlich gelenktes und politisches Projekt wird häufig abgegrenzt von Regionalisierung als marktgetriebenem oder ökonomischem Phänomen (Mansfield u. Milner 1999, S. 591). Zudem beschränken wir uns in diesem Artikel auf Regionalintegration geographisch $z u$ sammenhängender Länder und gehen damit auf die große Zahl von Freihandels- und präferenziellen Abkommen nicht weiter ein.

3 Im dritten Teil erläutern wir näher, was genau wir mit „Ähnlichkeiten“ meinen. 
Perspektive, die in den letzten Jahren verstärkt Eingang in die Politikwissenschaft und Internationale Beziehungen gefunden hat. Wir wollen dabei keineswegs bestehende vertikale Perspektiven gänzlich oder auch nur weitgehend durch horizontale ersetzen, sondern schlagen vielmehr vor, dass sie als sowohl empirisch fruchtbare wie theoretisch anschlussfähige zusätzliche Analyseperspektive stärker berücksichtigt werden sollten (ähnlich auch Jahn 2009 für die Vergleichende Regierungslehre).

Die generelle Stoßrichtung unserer Analyse und Forschungsagenda ist nicht neu. Bereits in den 1960er- und 1970er-Jahren, als eine erste Generation systematischer Analysen zur Regionalismusforschung entstand, zeigten mehrere Autoren die Rolle horizontaler Einflüsse auf. Nachdem Ernst Haas schon früh auf die Möglichkeit verwiesen hatte, dass andere Regionen den europäischen Einigungsprozess imitieren könnten (und sollten) (Haas 1961, S. 366), versuchten verschiedene Regionalismusexperten diesen „demonstration effect“ genauer zu untersuchen (Kaiser 1968, S. 95-96). So beschrieben ihn manche als „,chain reaction that induces unification in other parts of the world“ (Pinder 1968, S. 37); andere sprachen vom Phänomen des „extra-regional echoing“ (Avery 1973) oder allgemeiner von der Rolle externer „catalysts“ (Nye 1965). Allerdings blieben diese Forschungsansätze konzeptionell wie theoretisch unterentwickelt und waren empirisch unsystematisch. Als der globale Regionalismus spätestens Ende der 70er weltweit in die Krise geriet, verloren viele Autoren ihr Interesse an dem Thema. Angesichts der eingangs erwähnten Ähnlichkeiten zwischen Regionalorganisationen (empirischer Aspekt) und Fortschritten bei der Konzeptualisierung horizontaler Einflüsse (theoretischer Aspekt) ist es nun an der Zeit, dieses Thema erneut aufzugreifen.

Wir entwickeln unsere Argumente in drei Schritten. Im folgenden zweiten Teil rezensieren wir die neuere Literatur zur Regionalismusforschung, um zu zeigen, dass diese konzeptionell überwiegend von der Unabhängigkeit bestehender Regionalismusphänomene ausgeht bzw. Interdependenz einseitig als hegemoniale Beeinflussung konzeptualisiert. Im dritten Teil skizzieren wir eine alternative horizontale Analyseperspektive - die der Diffusion - und erörtern prominente Kanäle und Mechanismen des Diffusionsprozesses in Bezug auf die Vergleichende Regionalismusforschung. Im vierten Teil diskutieren wir anhand von drei zentralen Fragen der Vergleichenden Regionalismusforschung, welchen Beitrag eine solche Diffusionsperspektive leisten kann.

\section{Bestehende Analyseperspektiven in der neueren Regionalismusforschung}

Die neuere wissenschaftliche Literatur zum Regionalismus ist beträchtlich. Dies bedeutet, dass der folgende Literaturüberblick kaum der Vielfalt der bestehenden Literatur gerecht werden kann. Dies ist auch nicht der Anspruch. Vielmehr geht es darum, die Tendenz in der bestehenden Forschung herauszuarbeiten, verschiedene Fälle von Regionalismus vorwiegend als voneinander unabhängige Phänomene aus einer Top-down- oder Bottom-up-Perspektive zu erfassen und damit eher Varianz zu erklären. 


\subsection{Bottom-up-Perspektiven}

Das Gros der Literatur interpretiert Regionalismus als das Ergebnis spezifischer Interessen-, Institutionen- und/oder Identitätskonstellationen, die von unten nach oben arbeiten. In dieser Sichtweise wird Regionalismus hauptsächlich von politischen Prozessen innerhalb einer Region angetrieben. Er entwickelt sich unter der Bedingung, dass die entsprechenden Voraussetzungen in der Region gegeben sind (Collier u. Messick 1975). Diese Perspektive tendiert dazu, die Unterschiede zwischen Regionalismusprozessen zu betonen, die häufig als diverse, gar widersprüchliche Reaktionen auf globale wirtschaftliche Kräfte gedeutet werden (Coleman u. Underhill 1998; Dosch 1997; Hettne 1999; Mols 1996; Nabers u. Ufen 2005).

Interessen-basierte Analysen interpretieren Regionalismusprozesse gemeinhin als das Ergebnis von Aushandlungsprozessen egoistischer Akteure innerhalb einer Region. Intergouvernementale Theorien regionaler Integration betonen die zentrale Rolle der Regierungen mächtiger Mitgliedsstaaten, die Regionalismus vorantreiben, um ihre geopolitischen Interessen oder die ökonomischen Belange ihrer Wähler zu schützen (Hoffmann 1966). In anderen Regionen heben Forscher in ähnlicher Weise die Verteilung von Interessen und Macht zwischen den Mitgliedsstaaten hervor, um Unterschiede im regionalen Institutionalisierungsgrad zu begründen (Grieco 1997; Schirm 2002). Liberale Autoren heben insbesondere auf den unterschiedlichen Einfluss nationaler Koalitionen ab, um die Dynamiken des Regionalismus zu erklären (Moravcsik 1998; Solingen 2008).

Institutionen-basierte Theorien in der Vergleichenden Regionalismusforschung stellen die Rolle nationaler oder regionaler Institutionen heraus, die die Verwirklichung von staatlichen Interessen je nach Grad der Supranationalisierung differentiell einschränken und dadurch Ergebnisse beeinflussen. Viele der Arbeiten zum europäischen Integrationsprozess haben solche Argumente in Bezug auf die Rolle supranationaler Institutionen gemacht (etwa Sandholtz u. Stone Sweet 1998). Anderswo sind institutionelle Argumente aufgrund der relativen Schwäche regionaler Institutionen weniger populär (Malamud 2005). Stattdessen haben Forscher die Rolle nationaler Institutionen wie Vetopunkten oder Demokratie untersucht, um die Dynamiken des Regionalismus zu erklären (Mansfield et al. 2008).

Konstruktivistische Arbeiten konzentrieren sich auf die Rolle von Ideen, kultureller Traditionen oder Identitäten. Diese Literatur hebt auf Differenzen in den Ideen über Regionalismus ab, um dadurch Varianz im Grad der Institutionalisierung zu erklären. Am weitesten fortgeschritten ist hier der Vergleich zwischen Asien und Europa, für die die Rolle von Identität betont wird, um die informelle, konsensuale und wenig institutionalisierte Form des Regionalismus in Asien zu erklären (Acharya 2009; s. auch unten). In einer der wenigen wirklich komparativen Arbeiten in diesem Feld hat Francesco Duina die Rolle von nationalen Rechtskulturen betont, um die unterschiedlichen Herangehensweisen an Wirtschaftsintegration zu erklären (Duina 2006). Andere Forscher haben Argumente zur Rolle von Ideen und Identitäten in der Entstehung regionaler Sicherheitsgemeinschaften vorgebracht (Kupchan 1996). 
Trotz ihrer epistemologischen, ontologischen und theoretischen Vielfalt teilen Forscher in dieser Gruppe die Annahme, dass Regionalismus primär von bottomup-Faktoren getrieben wird und dass die jeweilige institutionelle Ausgestaltung von Regionalorganisationen somit durch Faktoren determiniert ist, die regional endogen sind und entsprechend große Variation aufweisen. Obwohl externe Einflüsse und weitere globale Trends nicht gänzlich vernachlässigt werden, üben sie ihren kausalen Einfluss nur aus, indem sie durch Akteure und Institutionen im nationalen oder regionalen Kontext kanalisiert und interpretiert werden. Diese Perspektive impliziert somit den konzeptuellen Schritt, dass Regionalismusprozesse als unabhängig voneinander betrachtet werden. Wir argumentieren dagegen, dass sich Prozesse der regionalen Institutionalisierung oftmals gegenseitig beeinflussen. Und obwohl wir die generelle Einschätzung teilen, dass Regionalorganisationen sich in ihrem Design unterscheiden, sind wir skeptischer gegenüber der Auffassung, dass ihr Design radikal variiert (vgl. Jetschke 2010).

\subsection{Top-down-Perspektiven}

Ein zweites Set an Analyseperspektiven stellt auf jene Faktoren ab, die Regionalismusprozesse von außen beeinflussen. Sie konzentrieren sich auf top-down-Faktoren, die den Entscheidungsspielraum von Regierungen einschränken und somit regionale Ergebnisse beeinflussen. Wir unterscheiden zwei Gruppen von Forschungsliteratur: eine beschäftigt sich mit globalen Wirtschaftsstrukturen, die andere mit der Rolle hegemonialer Akteure.

Erstere, dominiert von Ökonomen, interpretiert Regionalismus als die rationale Antwort politischer Akteure auf strukturelle Veränderungen in der Weltwirtschaft - Globalisierung - die Anreize für politische Strategien bieten, welche wiederum die Möglichkeiten wachsender ökonomischer und finanzieller Interdependenz nutzen (Lawrence 1996). Regionalismus hilft Staaten, Skaleneffekte in größeren regionalen Märkten zu schaffen und zieht ausländische Direktinvestitionen an (Büthe u. Milner 2008). Die zentrale zugrunde liegende Annahme ist daher, dass die Expansion des Regionalismus seit Ende des Kalten Krieges eine gemeinsame, jedoch unabhängige Reaktion auf ähnliche strukturelle Entwicklungen in der Weltwirtschaft darstellt. Zudem beschäftigt sich die Literatur hierzu primär mit der Frage, ob Regionalismus der globalen Wirtschaftsintegration, auch in Form multilateraler Handelsliberalisierung, zuträglich oder abträglich ist; weniger damit, welche Dynamiken verschiedene Formen des Regionalismus beeinflussen (siehe Bhagwati 1994).

Ein zweiter Strang der top-down-Literatur interpretiert Regionalismus als stark von Hegemonen beeinflusst, die solche Prozesse aufgrund eigener strategischer (Wirtschafts-) Interessen beeinflussen und koordinieren. Nach dem Ende des Kalten Krieges verfolgten sowohl die USA wie auch die EU zunehmend regionale Handelsstrategien, die nicht nur das multilaterale Handelssystem schwächten, sondern auch zur Entstehung neuer oder zur Veränderung bestehender Integrationsprojekte beitrugen (Bhagwati 1994; Telò 2001). Einige Autoren haben die Effekte unterschiedlicher ,Hegemonialstrategien“ in derselben Region, insbesondere in Lateinamerika, analysiert (Dimon 2006; Grugel 2004; Sbragia 2002). Andere 
betonen hingegen die Variation hegemonialer Strategien über Regionen hinweg und erklären damit Unterschiede im Design von Regionalorganisationen. Insbesondere Peter Katzenstein argumentiert, dass die Varianz in Institutionalisierungsgraden zwischen Asien und Europa auf unterschiedliche Strategien (Multilateralismus vs. Bilateralismus) der US-Außenpolitik zurückzuführen ist (Katzenstein 2005). Statt auf hegemoniale Akteure als solche abzustellen, hat eine andere Gruppe von Forschern den Einfluss von deren Strategien auf die Empfängerseite betrachtet. Sie hat die verschiedenen Formen des Widerstands und dessen Folgen als Reaktion auf die Verfolgung von ,Hegemonialstrategien' untersucht (Bowles 2002). Mark Beeson hat den Begriff „reactionary regionalism“ geprägt, um die Dynamik im ostasiatischen Regionalismus als einen Versuch, den Einfluss der USA zu mildern, zu fassen (Beeson 2003).

Diese bestehenden Analyseperspektiven haben wichtige empirische und theoretische Einsichten hervorgebracht, sie bleiben unserer Meinung nach jedoch unvollständig: Die Literatur zur Globalisierung geht in erster Linie der Frage nach, ob Regionalismus ein Stolperstein oder ein Baustein für ein globales Freihandelsregime ist oder ob Prozesse der Konvergenz oder Divergenz nationalstaatlicher Politik zu erwarten sind (Coleman u. Underhill 1998; Sampson u. Woolcock 2003). Das konkrete Design von Regionalorganisationen steht nicht im Zentrum der Analyse. Ansätze der hegemonialen Koordination vermuten die Ähnlichkeit von Regionalorganisation als das Ergebnis der (zwangsweisen) Durchsetzung durch einen Hegemon. Sie vernachlässigen, dass Mitglieder von Regionalorganisationen freiwillig bestimmte Institutionen oder Politiken übernehmen könnten, weil sie sie für nützlich oder angemessen halten (vgl. auch Acharya 2009). Hier kann die Diffusionsperspektive, die Regionalismusprozesse als interdependente Phänomene begreift, dazu beitragen, neue Forschungsfragen aufzuwerfen und althergebrachte neu zu beantworten.

\section{Diffusion als Horizontalperspektive in der Regionalismusforschung}

In diesem Abschnitt diskutieren wir zunächst eine Diffusionsperspektive auf Regionalismus und gehen dann auf prominente Diffusionskanäle und -mechanismen ein. Die Erforschung von Diffusionsprozessen ist seit den 1960er-Jahren in der Vergleichenden Politikwissenschaft fest verankert und hat zunehmend Eingang in die internationale Politik gefunden (Elkins u. Simmons 2005; Holzinger et al. 2007; Katz et al. 1963; Simmons et al. 2006). Diffusion wird dabei unterschiedlich definiert. Howard Aldrich (1979, S. 44) etwa spricht von der „Übernahme von innovativen Aktivitäten und Strukturen über Populationen hinweg “ und Everett M. Rogers (1983, S. 5-6) definiert Diffusion als „spontane, ungeplante Verbreitung neuer Ideen ", die sich vor allem durch Kommunikationskanäle vollzieht und Informationen über die Effektivität von Politiklösungen transportiert. In den Internationalen Beziehungen wiederum stehen Prozesse von Interdependenz im Vordergrund. Most und Starr (1990, S. 391) etwa sehen Diffusion als die „Ansteckungswirkung bestimmter Ereignisse" (contagion of events) in Form von Dominoeffekten, während Elkins und Simmons (2005, S. 33, 35) Diffusion als „Anhäufung ähnlicher Entscheidungen“ definieren, die „durch unkoordinierte 
Interdependenz" gekennzeichnet ist. Trotz ihrer unterschiedlichen Schwerpunkte und spezifischen Ausrichtungen (zumeist abhängig vom jeweiligen Forschungsgegenstand) haben diese Definitionen zwei Elemente gemein, die als Kern der Diffusionsperspektive charakterisiert werden können. Erstens bezeichnen sie einen Ausbreitungsprozess, der in temporaler oder geographischer Hinsicht geballt auftritt. In diesem Zusammenhang ist oft von „Wellen“ oder „Clustern“ als den prägnantesten Ausprägungen von Diffusion die Rede (etwa Elkins u. Simmons 2005; Walker 1969). Zweitens beziehen sie sich auf ein Phänomen, das dezentral abläuft, d. h., in dem eine Vielzahl unterschiedlicher Akteure in Abwesenheit einer zentral koordinierenden Instanz freiwillig ähnliche Entscheidungen treffen. ${ }^{4}$ Analytischen Mehrwert, zumal im Kontext der Vergleichenden Regionalismusforschung, bietet die Diffusionsperspektive unserer Ansicht nach nur dann, wenn sie in dieser Form enger gefasst und nicht als allumfassendes Konzept zur Bezeichnung jeglicher Ausbreitung von Institutionen oder Politiken genutzt wird, also auch solche einschließt, die durch hegemoniale Koordination zustande kommt. Dies hat nicht nur Auswirkungen für die mit dieser Perspektive verbundenen Mechanismen (Abschnitt 3.1), sondern auch für die sich daraus ergebende Forschungsagenda (Abschnitt 4).

Mit Elkins und Simmons gehen wir insofern davon aus, dass Diffusion als interdependente Entscheidungssituation konzipiert werden kann, bei der Entscheidungen auf der Ebene von Regionalorganisationen nicht unabhängig von Entscheidungen in anderen Regionalorganisationen getroffen werden, sondern sich Entscheidungsträger wechselseitig beobachten und entsprechend reagieren. Diese Reaktion drückt sich oft in der Übernahme ähnlicher Institutionen und Politiken durch die Regionalorganisationen aus. Die Übernahme von Regionalismusmodellen kann sich auf verschiedene Entscheidungsebenen beziehen: Auf einer Institutionenebene (Polity) beobachten wir, dass Staaten Regionalorganisationen nicht auf dem Reißbrett neu entwickeln, sondern sich an den institutionellen Designs anderer Organisationen orientieren. Auf regionaler Ebene finden wir sowohl institutionelle Kopien der EU, wie beispielsweise den Gemeinsamen Markt für das Östliche und Südliche Afrika (COMESA) oder die Andengemeinschaft, als auch der EFTA (beispielsweise die ASEAN und CARIFTA in den 1960er-Jahren) und zudem Kopien von Kopien, wie beispielsweise die Südasiatische Vereinigung für Regionale Kooperation (SAARC) als Kopie der ASEAN (Dash 1996). Entscheidungsträger in anderen Regionen kopieren manchmal nur einzelne Institutionen der EU, so etwa das Modell des EuGH oder das Europäische Parlament (Dri 2009). Auf einer Politikprogrammebene (Policy) beobachten wir, dass Regionalorganisationen ähnliche Politiken entwickeln wie beispielsweise in der Wettbewerbspolitik der Schaffung integrierter Märkte („single market“) oder bei vertrauens- und sicherheitsbildenden Maßnahmen (Katsumata 2010). Schließlich beobachten wir auf einer Politikprozessebene (Politics), dass Regionalorganisatio-

4 Freiwilligkeit sollte hier nicht als die Abwesenheit jeglicher constraints in einer Entscheidungssituation verstanden werden, sondern bezieht sich auf das Fehlen direkten externen Drucks eines identifizierbaren Akteurs. 
nen ähnliche Interessengruppen befördern oder ähnliche Entscheidungs- und Abstimmungsmechanismen schaffen (Bicchi 2006).

Aus einer politikwissenschaftlichen Perspektive sind diese Phänomene hochinteressant. Sie legen nahe, dass „nicht funktionale Elemente der einzelnen Untersuchungseinheiten für die Erklärung von Variation in den zu erklärenden Phänomenen verantwortlich sind, sondern vielmehr Einflüsse, die jenseits der Einzelelemente zu suchen sind und sich in Form von Diffusion auf die zu erklärende Variable bemerkbar machen“ (Jahn 2009, S. 94). Auf die Erklärung der Entstehung von Regionalorganisationen und ihres institutionellen Designs angewandt bedeutet das nichts anderes, als dass Erklärungen die Effekte anderer Regionalorganisationen berücksichtigen müssen. Erklärungstheoretisch besteht die Herausforderung einer Diffusionsperspektive also darin, die Regionalismusphänomene, die das Ergebnis interdependenter Prozesse und gegenseitiger Einflussnahme sind, von denen zu unterscheiden, die eine unabhängige Anpassung an interne Gegebenheiten darstellen („Galton's Problem“; siehe Franzese u. Hays 2008, S. 574-575). Daraus ergibt sich, dass der Prozessdimension bei der Diffusionsperspektive eine besondere Bedeutung zukommt, sowie eine funktionalistische oder unabhängige Erklärung als Nullhypothese getestet werden muss (etwa Simmons et al. 2006).

Wann ähneln sich Regionalorganisationen? Angesichts der Tatsache, dass nahezu keine Regionalorganisation außerhalb Europas (und nicht einmal Regionalorganisationen in Europa) einen so weitgehenden Souveränitätstransfer unternommen hat, wie wir ihn in der EU beobachten, stellt sich die Frage, wann wir von Ähnlichkeit mit der EU oder Ähnlichkeit anderer Regionalorganisationen untereinander sprechen, wenn die entsprechenden Funktionen nicht repliziert werden. Manche Institutionen haben einen hohen Erkennungswert, wie beispielsweise der Europäische Gerichtshof, der durch die direkte Klagemöglichkeit, die Direktwirkung und den Vorrang des Gemeinschaftsrechts eindeutig bestimmbar ist und auch so in anderen Regionalorganisationen wieder auftaucht (Alter u. Helfer 2010). Was aber zeichnet das institutionelle EU-Design aus? Was charakterisiert im Verhältnis dazu die EFTA? Das Charakteristikum der EU ist zweifelsohne der weitreichende Souveränitätstransfer, der sich in den Kompetenzen der Kommission und des Gerichtshofes sowie des Europäischen Parlaments ausdrückt. Wir sprechen dann von Ähnlichkeit zwischen Regionalorganisationen, wenn Staaten mehr oder weniger das gesamte institutionelle Design übernehmen, wir also Ähnlichkeiten in der institutionellen Geometrie vorfinden. Die für die EU typischen Institutionen sind eine Kommission mit supranationalen Kompetenzen im Bereich der wirtschaftlichen Integration, ein Europäischer Rat (der Regierungschefs), ein Ministerrat in verschiedenen Zusammensetzungen, ein Gerichtshof und ein regionales Parlament. Rat und Kommission sind jeweils funktionale Komitees angelagert, wobei sich diese bei der EU dadurch auszeichnen, dass sie ihren Sitz zentral in Brüssel haben. Im Vergleich dazu verfügte die EFTA bis 1994 weder über einen Gerichtshof noch über eine Kommission. Die Kommission ist in der EFTA das Generalsekretariat, das mit weit weniger Kompetenzen ausgestattet ist. EFTA hat die funktionalen Komitees dezentral in den Mitgliedsstaaten angesiedelt. Wir sprechen aber auch dann von Ähnlichkeit, wenn Staaten den Souveränitäts- 
transfer nicht oder nur zum Teil vollziehen. Und wir sprechen von Diffusion, wenn Regionalverbünde einzelne Institutionen der EU übernehmen (d. h. inklusive supranationaler Kompetenzen), wie den Gerichtshof. Wir wählen hier bewusst ein relativ weitmaschiges Kriterium und lassen zunächst die konkreten Funktionen, die die jeweiligen Institutionen erfüllen, unberücksichtigt, insbesondere, was den Souveränitätstransfer anbelangt, auch wenn wir uns der Probleme, die damit verknüpft sind, durchaus bewusst sind. Vor allem aus einer vergleichend-politikwissenschaftlichen Perspektive ist diese Definition von Ähnlichkeit kritikwürdig. Einige Regionalorganisationen wie beispielsweise die CARICOM haben zwar bei ihrer Weiterentwicklung aus der CARIFTA das institutionelle Gefüge der damaligen EG übernommen, nicht aber den Souveränitätstransfer vollzogen. Ist die CARICOM deshalb ähnlich zur EU? Wir argumentieren: ja. Uns interessiert Transfer, auch wenn Funktionen nicht ähnlich sind, weil wir davon überzeugt sind, dass die Frage nach gleichen Funktionen den Blick auf das interessante Phänomen der Diffusion an sich verstellt.

In gewisser Hinsicht bauen beide Seiten, Diffusionsforschung und Vergleichende Politikwissenschaft, an diesem Punkt Strohmänner auf. Aus einer Diffusionsperspektive vergrößert die Vergleichende Regionalismusforschung möglicherweise kleine Unterschiede, um erklärungsbedürftige Varianz zu erzeugen. Aus der Perspektive der Vergleichenden Politikwissenschaft nivelliert Diffusionsforschung diese Unterschiede weitgehend, um von Ähnlichkeit sprechen zu können. ${ }^{5}$ Aus unserer Perspektive ist eine Anpassung der übernommenen Institutionen an die Präferenzen der übernehmenden Staaten, beispielsweise in Bezug auf den Souveränitätstransfer, Teil von Diffusionsprozessen und eröffnet weitere Fragestellungen; beispielsweise die Frage, welche Faktoren beeinflussen, ob ein Souveränitätstransfer gleich, später oder gar nicht vollzogen wird. Aber Anpassung selbst sollte nicht direkt zum Ausschlusskriterium für Diffusion werden (vgl. dazu auch Avery 1973). Würde man das tun, könnte man eine der spannendsten Fragen gar nicht stellen: Warum geben Staaten ihren Regionalorganisationen überhaupt ein Design, dass der EU oberflächlich sehr ähnelt, wenn sie den Institutionen dann ganz andere Kompetenzen verleihen? Ist das Teil eines großen Missverständnisses, etwa weil Gründungsverträge oftmals regelrecht kopiert werden, wie im Fall der CARIFTA, dort aber in den wenigsten Fällen die genauen Funktionen erklärt sind? Oder ist der Schein tatsächlich bedeutend, etwa weil sich damit Anerkennung und Legitimation verbinden? Außerdem erzeugt - wie wir gerade gesehen haben - diese Vorgehensweise im zweiten Schritt die Varianz, die für die Vergleichende Politikwissenschaft wieder interessant ist. Was erklärt, wann Staaten sich dann entscheiden, auch die Funktionen zu übernehmen? Zuletzt möchten wir auf andere Forschungsfelder verweisen, die ebenfalls davon profitiert haben, Varianz zugunsten einer Anhäufung ähnlicher Prozesse zu minimieren, selbst wenn es um komplexe Institutionen geht, wie beispielsweise die Demokratieforschung (vgl. Huntington 1991; O‘Loughlin et al. 1998).

5 Wir danken einem Gutachter ausdrücklich für diesen Hinweis. 
Mit dieser Entscheidung eröffnet sich unserer Meinung nach auch die Möglichkeit, soziologische Diffusionsansätze stärker als mögliche Erklärung zu berücksichtigen und die von diesen Ansätzen thematisierten Entkopplungseffekte näher zu untersuchen (vgl. etwa Meyer u. Rowan 1977). Darunter verstehen die Autoren, dass - in ihrer Terminologie - Organisationen, die das Design anderer angesehener Organisationen emulieren, formale Strukturen und Aktivitäten erfolgreich voneinander trennen, um lediglich den Schein einer ,erfolgreichen Organisation aufrechtzuerhalten.

\subsection{Diffusionskanäle}

Angesichts der zentralen konzeptuellen Annahme der Interdependenz von Regionalorganisationen muss ihre gegenseitige Beeinflussung den analytischen Kern der Diffusionsperspektive bilden. Ein überzeugendes Argument dieser Art sollte daher die Kanäle identifizieren, durch die Informationen und Praktiken diffundieren und die Logik explizieren, durch die sie ihren kausalen Einfluss ausüben. Die neuere Regionalismusforschung bietet hier eine Vielzahl an empirischen Phänomenen, die wir mit dem Konzept der Diffusion erfassen und neu bewerten können und die interessante Anknüpfungspunkte an andere, derzeit wachsende Forschungsfelder bieten. In diesem Abschnitt unterscheiden wir drei Arten von Kanälen - virtuelle, informelle und formalisierte -, die unterschiedliche Charakteristika in Bezug auf die relevanten Akteure, deren ,Diffusionsinstrumente' und ihre Wirkungen auf Regionalintegration aufweisen und daher unterschiedliche Forschungsfragen aufwerfen. In der Realität treten sie jedoch oft gemeinsam auf.

\subsubsection{Virtuelle Kanäle: Kommunikationstechnologien}

Virtuelle Diffusionskanäle bezeichnen die Übertragung relevanter Informationen zwischen regionalen Entscheidungsträgern mithilfe moderner Kommunikationsund Informationstechnologien. Entscheidungsträger können sich dabei aktiv auf die Suche nach solchen Informationen begeben, indem sie etwa das Internet nach Informationen durchsuchen, um den eigenen Integrationsprozess voranzutreiben. In der Regionalismusforschung hat Francesco Duina etwa dokumentiert, dass regionale Entscheidungsträger Formen der Regulierung oder Verrechtlichung in anderen Regionen über das Internet studieren und dass dies häufig zur mehr oder weniger vollständigen Übernahme der entsprechenden Herangehensweisen führt (Duina 2006, S. 96). Jedoch fehlt uns ein systematischeres Bild von der Bedeutung solcher Prozesse und den Faktoren, die sie beeinflussen: Welche Art von Information wird besonders gesucht und aus welcher Quelle? Unter welchen Bedingungen suchen Entscheidungsträger solche Informationen und mit welchen Folgen?

Andererseits berichten Massenmedien zunehmend über Integrationsprozesse in anderen Teilen der Welt, insbesondere über Vorgänge in der EU. Verschiedene Forschungsprojekte haben begonnen, die Wahrnehmung der EU außerhalb Europas zu erfassen, beispielsweise durch eine systematische Analyse der Medienberichterstattung über die EU (Lucarelli 2007). Solche Medienberichterstattung 
spielt jedoch nicht nur eine Rolle in Bezug auf die Wahrnehmung der EU als internationalem Akteur. Sie ist auch aus Sicht einer Diffusionsperspektive interessant. Massenmedien fungieren als ,Transmissionsriemen' für Informationen zu Integrationsprozessen in anderen Teilen der Welt, die auch unabhängig von der aktiven Suche durch Entscheidungsträger Effekte hervorrufen können. Wir vermuten, dass die Medienberichterstattung über den europäischen Integrationsprozess die Wahrnehmung von Entscheidungsträgern in anderen Teilen der Welt beeinflusst. Je häufiger über die EU berichtet wird und umso positiver die Berichterstattung ist, desto größer ist die Wahrscheinlichkeit der Übernahme durch andere Organisationen. Entscheidungsträgern in Regionalorganisationen oder innenpolitischen Interessengruppen werden dadurch innovative institutionelle Lösungen für funktionale Probleme der eigenen Regionalorganisation näher gebracht, und zwar unabhängig von Politiknetzwerken (s. nächster Abschnitt), die eine Lösung befördern. Es wäre beispielsweise interessant zu untersuchen, ob die Wahl des Designs einer Regionalorganisation neben anderen Faktoren auch mit dem Ausmaß der Berichterstattung über konkurrierende Regionalorganisationen korreliert. Beispielsweise gab es in den 1960er-Jahren nicht nur die EG als Modell regionaler Kooperation, sondern alternative Regionalorganisationen wie den Nordischen Rat (1952), der ebenfalls Nachahmer wie das lateinamerikanische Parlatino (1964) hatte (Marschall 2005).

\subsubsection{Informelle Kanäle: Transnationale Netzwerke}

Informelle Kanäle beziehen sich auf die direkte, nicht-institutionalisierte Interaktion zwischen staatlichen und nicht-staatlichen Akteuren, insbesondere in Form transnationaler Netzwerke. Die Verbreitung und der wachsende Aktivismus solcher Netzwerke sind bereits für eine Vielzahl von Politikbereichen dokumentiert und analysiert worden. Es gibt daher wenig gute Gründe, warum sie im Bereich des Regionalismus weniger weit verbreitet und weniger einflussreich sein sollten, insbesondere vor dem Hintergrund, dass Integrationsprozesse sich weltweit ausbreiten und vertiefen. Solche transnationalen Experten-Netzwerke beeinflussen Entscheidungsträger in einer Vielzahl von Politikfeldern. Sie agieren als Träger von Ideen und Wissen, ohne formal politische Macht zu besitzen, und müssen durch Expertise und gute Argumente überzeugen. Damit sind Einflussmechanismen bezeichnet, die charakteristisch für Horizontalperspektiven sind (Haas 1992).

In der Tat gibt es erste empirische Belege, die auf ihre wachsende Existenz und Bedeutung hinweisen. Erstens haben Forscher intra-regionale Politiknetzwerke und ihren Einfluss in bestimmten Regionen analysiert. Brian Jobs (2003) Arbeiten zum Beispiel befassen sich mit der Rolle nicht-staatlicher Netzwerke im asiatischen Regionalismus wie der ASEAN-ISIS oder verschiedener Eminent Persons Groups (EPGs). Mariana Botto untersucht die Rolle akademischer Netzwerke im lateinamerikanischen Regionalismus (Botto 2009). Zweitens haben Forscher angefangen, die Rolle epistemischer Gemeinschaften und ,advocacy coalitions ' bestehend aus Aktivisten bestimmter Berufsgruppen zu untersuchen. Karen Alter 
etwa untersucht die Rolle transnationaler „jurist advocacy networks“ bei der Entstehung und Funktionsweise des EuGH und des Andengerichtshofs (Alter 2008). Osvaldo Saldías stellt einen nahezu exemplarischen Einfluss transnational agierender Juristen bei der Übernahme des EuGH durch die Staaten der Andengemeinschaft fest. Diese waren über das Institute for the Integration of Latin Amer$i c a$ and the Caribbean und die Inter-Amerikanische Entwicklungsbank vernetzt: "[T]he drafters [...] used mainly European informational resources to look for a solution and to shape their proposal" (Saldías 2010, S. 8). Andere wiederum studieren die Rolle regionaler Wirtschaftsorganisationen wie der asiatischen Entwicklungsbank oder der Wirtschaftskommission für Lateinamerika in Regionalismusprozessen (Dent 2008). Drittens gibt es einige Darstellungen über die Unterstützung von EU-orientierten epistemischen Gemeinschaften durch die Europäische Kommission. In der Regionalintegration finanziert diese Trainings- und Forschungszentren wie die Asien-Europa Stiftung (ASEF) in Singapur, das Europäische Institut für öffentliche Verwaltung (EIPA) in Maastricht, das Trainingszentrum für Regionalintegration (CEFIR) in Montevideo oder das Institut für europäisch-lateinamerikanische Beziehungen (IRELA) in Madrid, die explizit das Ziel verfolgen, Integrationserfahrungen zu transferieren (Sanchez-Bajo 1999, S. 935).

Wir vermuten, dass es sich dabei um einflussreiche Agenten horizontalen Einflusses im globalen Regionalismus handelt. Wir wissen aber nicht, wie viele dieser Netzwerke es gibt, wann sie entstehen oder wie sie ihren Einfluss in diesem Bereich geltend machen. Und wir wissen sehr wenig über die Randbedingungen, unter denen sie besonders einflussreich sind.

\subsubsection{Formalisierte Kanäle: Interregionale Beziehungen}

Interregionale Beziehungen sind der wichtigste Kanal für die formalisierte Interaktion zwischen Regionalorganisationen. Entstanden mit dem Ziel, Regionen dabei zu unterstützen, mit grenzüberschreitenden Kooperationsproblemen umzugehen, haben sie sich seit dem Ende des Kalten Krieges stark ausgeweitet (Gilson 2005; Hänggi et al. 2006; Rüland 2010). Die EU, die Beziehungen mit Regionen denen mit Einzelstaaten vorzieht, hat diese Entwicklung maßgeblich vorangetrieben (Edwards u. Regelsberger 1990; Söderbaum u. van Langenhove 2006). Während die EU 1975 erst zwei solcher formalisierten interregionalen Beziehungen unterhielt, waren es 1990 bereits zwölf und im Jahr 2007 insgesamt 29 (vgl. Alecu de Flers u. Regelsberger 2005, S. 321; Smith 2008, S. 103-104). ${ }^{6}$

Interregionale Beziehungen sind normalerweise gut institutionalisiert mit einer Bandbreite an Foren für regelmäßige Kontakte zwischen Entscheidungsträgern auf verschiedenen Ebenen, manchmal unter Beteiligung der Zivilgesellschaft (Alecu de Flers u. Regelsberger 2005). Wir können zwei Arten von Rückkopplungseffekten solcher Beziehungen für Regionalismus auf beiden Seiten unterscheiden, die sich mit der Konzeption von Institutionen als Förderern und Orten für be-

6 Interregionale Beziehungen sind jedoch auch in der Süd-Süd Kooperation stark gewachsen (etwa Trivedi 2006). 
stimmte Effekte decken (Checkel 2005, S. 806-807). Erstens kann die schiere Existenz institutionalisierter Interaktion zwischen Regionalprojekten einen strukturellen Einfluss auf ihre jeweilige Kohäsion haben. Eine Studie argumentiert, dass solche Interaktionen die interne Kapazität von Regionalprojekten erhöhen kann (Doidge 2007; siehe auch Hänggi 2007). Zweitens kann der regelmäßige Kontakt in solchen Foren Sozialisierungs- und Überzeugungseffekte in Bezug auf die beteiligten Akteure haben (Yeo 2008). Interregionale Beziehungen stellen daher wichtige Kanäle des horizontalen Einflusses auf Regionalismus dar, die die Literatur bisher fast vollständig vernachlässigt hat (Ausnahmen sind Farrell 2007; Lombaerde u. Schulz 2009). Dennoch sind sie ein Erfolg versprechender Gegenstand für zukünftige Forschung: Unter welchen Bedingungen und in welcher Art und Weise beeinflusst Interregionalismus Regionalismus? Gibt es bestimmte Bereiche des Regionalismus (Problemfelder, Entscheidungsebenen, Politikbereiche etc.), die stärker davon betroffen sind, und wenn ja, warum? Führt Interregionalismus zur Konvergenz zwischen regionalen Integrationsmodellen oder kann er auch den gegenteiligen Effekt haben? Und wie und mit welchen Ergebnissen interagieren verschiedene Diffusionskanäle?

\subsection{Mechanismen der Diffusion}

Eine zentrale Forschungsfrage von Diffusionsstudien in den letzten Jahren war die nach der Definition, Konzeptualisierung und Analyse der Kausalmechanismen, über die sich Institutionen und Politiken ausbreiten. Entsprechend unseres Verständnisses von Diffusion als horizontaler Analyseperspektive schlagen wir vor, vertikale Mechanismen wie etwa Zwang oder materielle Anreize, zumindest im Kontext der Vergleichenden Regionalismusforschung, nicht als Diffusionsmechanismen zu definieren. In der Europäisierungsforschung (Börzel u. Risse 2009, 2011; Schimmelfennig u. Sedelmeier 2005) und in manchen IB-inspirierten Diffusionsstudien (etwa Simmons et al. 2006) spielen diese aus nachvollziehbaren Gründen eine zentrale Rolle, denn sie sind in der Tat wichtige Wege, durch die hegemoniale Akteure ihren Interessen entsprechende Modelle verbreiten. Diese Arten der Verbreitung sind jedoch in der Regionalismusforschung bereits ausreichend aus der Analyseperspektive der ,hegemonialen Koordination' theoretisch erfasst und vereinzelt empirisch untersucht worden (siehe Abschnitt 2.2). Analytisch bietet hier eine Diffusionsperspektive aus unserer Sicht kaum Mehrwert. ${ }^{7}$ Zudem ist insbesondere die institutionelle Konfiguration von Regionalorganisationen, deren Verständnis wir als primären Mehrwert der Diffusionsperspektive verstehen, wenn überhaupt nur sehr indirekt von Zwangs- und Anreizmechanismen beeinflusst. Die EU und USA haben die Wahl von regionalen Institutionen niemals direkt mit der Konditionalität von Handelsabkommen oder Entwick-

7 Selbst bei prominenten VertreterInnen der Diffusionsperspektive wird diese Unterscheidung nicht einheitlich gehandhabt. Elkins und Simmons (2005, S. 38) beispielsweise definieren Diffusion zuerst als horizontale Beeinflussungssituation „but not through any collaboration, imposition or otherwise programmed effort on the part of any of the actors“, präsentieren wenig später jedoch Zwang als zentralen Diffusionsmechanismus (Simmons et al. 2006). 
lungshilfszahlungen verbunden, wie es etwa für Menschenrechte oder Rule-ofLaw-Standards der Fall ist. Insofern schlagen wir vor, unter Diffusionsmechanismen in der Regionalismusforschung ausschließlich ,horizontale' Mechanismen zu fassen, die keine hierarchische (vertikale) Beziehung zwischen zwei klar identifizierbaren Akteuren voraussetzt.

Allerdings ist auch bei dieser ,Mechanismengruppe die begriffliche Vielfalt enorm und es bleibt manchmal unklar, ob die Unterschiede verschieden benannter Mechanismen rein semantischer oder auch konzeptioneller Natur sind. Sie können jedoch in Bezug auf die dahinter stehende Entscheidungslogik grob auf einem Kontinuum verortet werden, dessen beiden Pole der klassischen Unterscheidung von Logik des Konsequentialismus und Logik der Angemessenheit entsprechen (siehe Darstellung 1).

\section{Darstellung 1: Diffusionsmechanismen in der Regionalismusforschung}

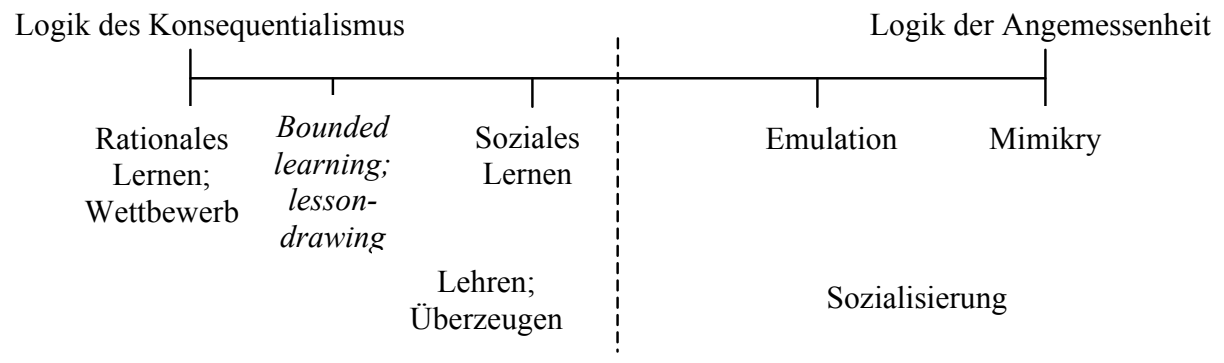

Auf der ,Konsequentionalismus'-Seite des Kontinuums liegt der Mechanismus des rationalen Lernens (,Bayesian updating'). Dabei suchen rational agierende Akteure, die sich an festgesetzten Zielen und Präferenzen orientieren, konsequent nach optimalen Lösungen, um den erwarteten Nutzen zu maximieren (Meseguer 2009; Sommerer 2011). Auf der anderen Seite des Kontinuums finden wir den Mechanismus der Mimikry, dem die Logik der Angemessenheit zugrunde liegt. In diesem Fall geht es weniger um die Suche nach effizienten Problemlösungen für ein bestehendes (Effizienz-) Problem, sondern es geht um die Suche nach Anerkennung, Glaubwürdigkeit und Legitimität. Soziologisch-institutionalistische Theorien gehen davon aus, dass Staaten ihrer Zugehörigkeit zu einer Staatengemeinschaft durch die Übernahme entsprechender Skripte expressiven bzw. symbolischen Ausdruck verleihen (Meyer u. Rowan 1977). So kann man argumentieren, dass es bei regionaler Integration nicht nur um Wohlfahrtsmaximierung und Effizienzsteigerung geht, sondern auch um die Verwirklichung von Wertvorstellungen wie Frieden und Freiheit. Ihnen liegt nicht unbedingt ein funktionales Problem zugrunde und entsprechend beobachten wir auch Übernahmen ohne funktionalen Problemdruck. Wir vermuten einen solchen Mechanismus in Fällen, in denen die Etablierung einzelner regionaler Institutionen oder ganzer Regionalorganisationen keine funktionale Logik erkennen lässt. Zum Beispiel bleibt ein ,Rätsel', warum es in den 1970er-Jahren zur Etablierung vieler Regionalparlamente kommt, selbst 
wenn die Regionalorganisationen selbst keinen Souveränitätstransfer vorsehen, der eine stärkere Kontrolle anzeigt, oder Mitglieder überwiegend autoritär sind.

Weitere horizontale Diffusionsmechanismen integrieren dann Aspekte der beiden Handlungslogiken und können entsprechend zwischen den Endpunkten angesiedelt werden. Andere Formen des Lernens, etwa bounded learning, lessondrawing oder soziales Lernen (Meseguer 2009; Hall 1993), folgen zwar nach wie vor primär der Logik des Konsequentialismus, enthalten jedoch Elemente der Angemessenheitslogik, etwa indem Entscheidungsträger sich primär auf Informationen und Lösungen von ,kulturell` ähnlichen oder nahestehenden Partnern stützen. Yeo (2008) sowie Jetschke und Murray (2011) zeigen etwa, wie Entscheidungsträger in der ASEAN EU-Modelle durch Lernprozesse übernommen haben. Ähnliches lässt sich für den Wettbewerbsmechanismus sagen, nach dem Staaten in dezentralisierter Form um Handelsmöglichkeiten und internationales Kapital miteinander konkurrieren. Wettbewerb führt dann zu Diffusionsprozessen, wenn Staaten bei anderen ,sehen', wie sie durch bestimmte Strategien wirtschaftlich erfolgreicher werden und daher ähnliche Strategien wählen. ${ }^{8}$ Mit abnehmendem Grad der angenommenen Rationalität nähert sich der Wettbewerbsmechanismus dem der Emulation an, der sich auf die Übernahme erfolgreicher Modelle primär aufgrund von Legitimitäts- oder Glaubwürdigkeitserwägungen bezieht. Lenz (2011) beleuchtet beispielsweise, unter welchen Bedingungen Entscheidungsträger in SADC und Mercosur institutionelle Arrangements der EU durch Emulationsprozesse übernehmen.

Diese horizontalen Mechanismen, die die jeweilige Empfängerseite betonen, finden ihre Entsprechung in ähnlichen Mechanismen, auf der Senderseite. Rationalistisch orientierte Lernprozesse verstehen unter lehren und überzeugen die Vermittlung eines besseren Verständnisses über kausale Ursache-Wirkungszusammenhänge (Haag 1992; Checkel 2005); auf Angemessenheit abstellende Übernahme entspricht dann Sozialisierungsprozessen (Finnemore 1993). ${ }^{9}$ Diese Mechanismen deuten darauf hin, dass Diffusionsprozesse von anderen Akteuren angeregt und unterstützt werden können. In unserem Kontext können solche Akteure sowohl innerhalb der Region angesiedelt sein, etwa in Form epistemischer Gemeinschaften, die Entscheidungsträger von bestimmten Lösungsansätzen zu überzeugen suchen, als auch außerhalb, etwa wenn EU-Akteure im politischen Dialog mit anderen Regionalorganisationen Sozialisierungsversuche unternehmen (siehe vorherige Besprechung der Diffusionskanäle). Wichtig ist jedoch zu betonen, dass diese Art der ,Einflussnahme‘ materielle Anreize oder Druck weitgehend ausschließt und somit nicht der Analyseperspektive der hegemonialen Koordination entspricht.

Hier stellt sich auch die Frage, inwiefern Diffusionskanäle und -mechanismen voneinander unabhängig sind oder nicht und ob sich entsprechende Hypothesen

8 Ansonsten haben Strategien zur Erhöhung der wirtschaftlichen Effizienz wenig mit dem Verhalten anderer Staaten zu tun (was häufig der Fall ist), fallen also nicht unter unsere Konzeption von Diffusion als interdependenter Entscheidungssituation.

9 Insofern geht es hierbei primär um die Frage, aus Perspektive welcher Akteure man den Diffusionsprozess beschreibt, nicht jedoch um die analytische Logik des Prozesses; diese bleibt gleich. 
über vermutete Zusammenhänge generieren lassen. Grundsätzlich könnte man spekulieren, dass die Übernahmen von Institutionen und Politiken aus anderen Regionalorganisationen bzw. die Geschwindigkeit dieser Übernahmen stark von der Dichte und Verteilung der Diffusionskanäle abhängen; die Grundlage jeder Netzwerkanalyse (vgl. Strang u. Meyer 1993). Bei sozial vernetzten Staaten sind grundsätzlich eher Übernahmen durch Lernprozesse zu vermuten, sei es in Politiknetzwerken oder interregionalen Foren. Politiknetzwerke verfügen vermutlich über eine größere Fähigkeit, über den direkten Austausch die ,Passgenauigkeit ${ }^{\star}$ der Lösungen zu diskutieren und forcieren damit Lernen. Medienberichterstattung würde es auch sozial isolierten Staaten ermöglichen, Informationen über regionale Integration zu verarbeiten, dieser Kanal kommuniziert aber Probleme höchstwahrscheinlich selten differenziert genug und könnte deshalb eher Mimikry provozieren. Interregionale Kanäle könnten durch ihre Exklusivität streng rationales Lernen behindern, da Akteure weniger geneigt sein werden, die Erfahrungen anderer Regionalorganisationen systematisch zu evaluieren. Aber auch Politiknetzwerke und interregionale Kanäle könnten Mimikry provozieren, nämlich dann, wenn sie Akteuren die Gelegenheit geben, ein gemeinsames Verständnis über die soziale Angemessenheit von regionaler Integration zu entwickeln. Selbst in Situationen, in denen regionale Integration nicht funktional notwendig erscheint, könnten Integrationsschritte dann nachvollzogen werden, eben weil sie als angemessen betrachtet werden. Abschließend wollen wir jedoch betonen, dass Hypothesen über den Zusammenhang von Diffusionskanälen und -mechanismen am sinnvollsten in Bezug auf konkrete Fragestellungen und die damit einhergehende Operationalisierung der jeweiligen Variablen generiert werden.

\section{Eine Diffusions-sensitive Forschungsagenda für die Vergleichende Regiona- lismusforschung}

Aus einer Diffusionsperspektive ergibt sich unserer Meinung nach eine innovative Forschungsagenda für die Vergleichende Regionalismusforschung, die sich an den drei traditionellen Kernfragen internationaler Institutionen im Allgemeinen und Regionalorganisationen im Besonderen orientiert. Sie bietet unserer Ansicht nach neue Antworten auf bestehende Fragen und kann dazu beitragen, die Rolle struktureller Faktoren im globalen Regionalismus neu zu bewerten oder die Handlungsmuster der beteiligten Akteure besser zu verstehen.

\subsection{Warum entstehen Regionalorganisationen?}

Die politikwissenschaftliche (und wirtschaftswissenschaftliche) Forschung argumentiert hier bisher primär rationalistisch-funktionalistisch. Generell entstehen Regionalorganisationen, ähnlich wie andere internationale Organisationen, unter Interdependenzbedingungen als Antwort auf Kooperationsprobleme oder wenn unilaterales Handeln suboptimale Ergebnisse erzielt (s. auch Keohane 1984). Dabei sind die Art der Kooperationsprobleme und die Verteilungsproblematik wichtige Determinanten für die Entstehung von Regionalorganisationen zur sicherheitspolitischen Kooperation (z. B. Afrikanische Union) oder der wirtschaftlichen 
Integration (NAFTA, EU, EFTA). Ein Rätsel für rationalistische Ansätze bleibt das Phänomen, warum wir die Entstehung und Ausbreitung von Regionalorganisationen in Gegenden beobachten, in denen Interdependenzen relativ gering sind, etwa in den meisten Gegenden des globalen Südens (Krapohl 2008).

Rationalistisch orientierte Autoren erklären die Existenz von Regionalorganisationen zwischen Staaten, die wenig wirtschaftliche Komplementarität aufweisen, als Versuch, die negativen Externalitäten der Wirtschaftsintegration im Norden durch eigene regionale Kooperation zu internalisieren (Mattli 1999). Baldwins (1993) „Dominotheorie“ des globalen Regionalismus teilt beispielsweise die zentrale konzeptionelle Annahme von Diffusionsanalysen, ohne dass er diesen Begriff explizit verwendet. Die Erklärung beruht jedoch in erster Linie auf Rationalitätsannahmen, nicht auf harter empirischer Evidenz. Es bleibt unklar, warum Entscheidungsträger in fast allen Teilen der Welt innerhalb kürzester Zeit sehr ähnliche Überlegungen angestellt haben sollen, obwohl deren wirtschaftliche Situationen sehr unterschiedlich waren und viele der Übereinkünfte primär auf dem Papier existieren, also kaum der effektiven Internalisierung von etwaigen Externalitäten dienen (s. unten).

Hier bietet die Diffusionsperspektive Ansätze für alternative Antworten und neue Fragen. Eine Perspektive, die wie Diffusion eine wechselseitige Beobachtung annimmt, könnte die wellenartige Ausbreitung von Regionalorganisationen nach dem 2. Weltkrieg erklären (Fawcett 1995): Die erste Welle erfolgte in den 1960erund 1970er-Jahren infolge der Gründung der EG, die zweite Welle nach dem Ende des Kalten Krieges infolge der endgültigen Schaffung des Gemeinsamen Marktes als Reaktion auf wachsenden globalen Wettbewerbsdruck (Sandholtz u. Zysman 1989). Die Gründung der NAFTA (1992) war bereits eine Reaktion auf die vertiefte europäische Integration im Rahmen der Einheitlichen Europäischen Akte, freilich unter anderen institutionellen Vorzeichen (Abbott 1992). Je mehr Länder sich Regionalorganisationen anschlossen, desto dringlicher schien es für andere Länder, dies ebenfalls zu tun. Eine solche ,Diffusionsinterpretation' wirft neue Fragen nach dem genauen Entstehungszeitpunkt von Regionalorganisationen und der damit verbundenen Motivation auf. Entstehen sie etwa unmittelbar, wenn innovative, Erfolg versprechende Modelle verfügbar werden, oder erst, wenn vergleichbare Probleme auftreten, die das Modell zu lösen vorgibt (s. Weyland 2008)? Kann die Verbreitung des Regionalismus in den frühen 90er-Jahren als einfaches Kopieren des neuen ,Modells' einer erfolgreichen Regionalorganisation in Zeiten erhöhten Wettbewerbsdrucks gelesen werden? Eine mögliche Erklärung für die Abwesenheit von Regionalismus in Nordostasien wäre dann, dass die wirtschaftliche Dynamik der Region einen vertieften Regionalismus zunächst nicht erforderte und die europäische Lösung des, Wettbewerbsproblems' durch formale Integration zwar erwogen, jedoch angesichts der bestehenden Netzwerkverbindungen zwischen den Volkswirtschaften verworfen wurde (Katzenstein 2005).

Aus einer Logik der Angemessenheit könnte die Entstehung von Regionalorganisationen außerhalb Europas damit erklärt werden, dass mit der EU ein Modell regionaler Kooperation entstanden ist, dass inzwischen zu einem Standard für angemessenes Verhalten in der Staatengemeinschaft avanciert ist. Je mehr Staaten 
diesem Standard folgen, desto attraktiver wird es für andere Staaten, sich ebenfalls einer Regionalorganisation anzuschließen, völlig unabhängig davon, ob dieses Verhalten wirtschaftlich rational ist oder eine besondere Aussicht auf Erfolg bietet. Aus dieser Perspektive ist Regionalintegration inzwischen zu einem integralen Bestandteil moderner Staaten geworden, der nicht mehr hinterfragt wird (Jolliff u. Jupille 2010). Im Zuge einer solchen Lesart gilt es etwa zu fragen, wie Regionalintegration diesen Status erreicht haben könnte und welche Nachteile Staaten haben, die diesem Standard nicht folgen. ${ }^{10}$ Die beobachtbare Implikation dieses theoretischen Zugriffs wäre, dass es einen signifikanten Unterschied zwischen der tatsächlich vorhandenen Zahl regionaler Zusammenschlüsse und ihrer erwartbaren Zahl angesichts der gegebenen wirtschaftlichen und sicherheitspolitischen Verflechtungen gibt.

\subsection{Was bestimmt die institutionelle Wahl / das Design von Regionalorganisa- tionen?}

Auch für das Design von internationalen Institutionen dominieren rationalistische Erklärungen. Das Forschungsprogramm des rationalen Designs internationaler Institutionen (RDII) geht davon aus, dass Designfragen endogen bestimmt sind, also durch die Zahl und Präferenzen der Staaten, deren Unsicherheit über zukünftige Entwicklungen oder deren Kooperationsprobleme (Koremenos et al. 2001). „Major institutions [are] organized in radically different ways“, was beispielsweise den Grad der Institutionalisierung, Mitgliedschaft oder Abstimmungsregeln betrifft (Koremenos et al. 2001, S. 761). Obwohl wir die generelle Einschätzung teilen, dass Regionalorganisationen sich in ihrem Design unterscheiden, sind wir skeptischer gegenüber der Größe der Varianz, die es unter internationalen Organisationen und insbesondere Regionalorganisationen gibt. Wie wir bereits oben anekdotisch gezeigt haben, gehen wir davon aus, dass die Ähnlichkeit in den institutionellen Designs größer als vermutet ist, und dass es ein beschränktes Menü an akzeptierten und effektiven Designs für Institutionen gibt, an dem sich Staaten orientieren. Die Andengemeinschaft nahm beispielsweise bei ihrer Suche nach einem effektiven Streitbeilegungsverfahren neben dem EuGH alternative Streitbeilegungsorgane unter die Lupe, wie diejenigen des Zentralamerikanischen Gemeinsamen Marktes, der Wirtschaftsgemeinschaft Westafrikas (ECOWAS) und des GATT: "[I]t was not intended by the working group to design a new dispute settlement mechanism from scratch" (Saldías 2010, S. 13). Die Gründungsmitglieder der Südostasiatischen Vereinigung (ASA), der Vorgängerorganisation der ASEAN, untersuchten zwei Regionalorganisationen, die EG und die EFTA, und entschieden sich dann dafür, das EFTA-Design zu übernehmen (Jetschke 2010). Die Frage ist dann aber, welche Faktoren diese Wahl bestimmen.

Grundsätzlich geht die RDII-Literatur davon aus, dass Staaten dasjenige Design wählen, welches ein bestehendes Kooperationsproblem am effektivsten löst. Dies setzt jedoch voraus, dass sie verschiedene Lösungen in Bezug auf ihre Effek-

10 Laut Statistik der WTO, ist die Mongolei beispielsweise das einzige Land der Welt, das keinem Freihandelsabkommen angehört. 
tivität unterscheiden können, was angesichts des Problems multipler Gleichgewichte problematisch sein kann (Goldstein u. Keohane 1993, S. 17). Warum wählen viele Regionalorganisationen ein EuGH-ähnliches Design für ihren Streitschlichtungsmechanismus, wenn Souveränitätsvorbehalte groß sind und mit dem WTO-Streitschlichtungsverfahren ein ähnlich effizienter Mechanismus zur Verfügung steht? In ähnlicher Weise lässt sich fragen: Ist die Schaffung und Stärkung eines Regionalparlamentes wirklich der effizienteste Weg, die Legitimitätsprobleme einer Organisation zu lösen (s. Goetze u. Rittberger 2010)?

Souveränitätserwägungen und Präferenzen innenpolitischer Gruppen spielen hierbei sicherlich eine Rolle für die Wahl eines bestimmten Designs (Moravcsik 1998). Aus einer Diffusionsperspektive rücken jedoch noch andere Variablen in den Fokus. Die koloniale Vergangenheit von Staaten etwa beeinflusst nicht nur Souveränitätserwägungen in wenig ,rationaler' Art und Weise - ein Zusammenhang, den Kahler (2000) herstellt -, sondern sie beeinflusst auch, wo Regierungsvertreter nach Vorbildern suchen. Außereuropäische ehemalige Kolonien waren zum Zeitpunkt der ersten Welle des Regionalismus oftmals bereits in institutionelle Wirtschaftsstrukturen eingebettet, die zum Teil noch aus der Kolonialzeit stammten, wie das Commonwealth unter Führung Großbritanniens. Koloniale Institutionen bzw. die durch sie kanalisierten Wirtschaftsstrukturen haben vermutlich großen Einfluss auf die Wahl des Designs. Großbritannien hat beispielsweise nach der Gründung der EFTA 1960 auch außerhalb Europas stark für diese Organisation geworben.

Koloniale Vergangenheit spielt auch aus einer Identitätsperspektive eine nicht zu unterschätzende Rolle. Die Schaffung von Regionalorganisationen in den 1960er- und 1970er-Jahren ist größtenteils ein Elitenprojekt (Busse 1999). Viele der Eliten in Ländern des Globalen Südens wurden jedoch in Europa sozialisiert und übernehmen von dort Ideen. So schreibt Thanat Khoman, der ehemalige thailändische Außenminister: "For many of us and for me in particular, our model has been and still is, the European Community, not because I was trained there, but because it is the most suitable form for us living in this part of the world - in spite of our parallel economies which are quite different from the European ones" (Khoman 1992, S. xix). Zudem scheinen bestimmte regionale Institutionen im Laufe der Zeit als ,normal' angesehen zu werden, sodass weniger genaue KostenNutzen-Abwägungen als vielmehr der Wunsch, der entstehenden ,Normalität‘ entsprechen zu wollen, die Wahl einer Institution bestimmt. So haben sich etwa regionale Parlamente in Lateinamerika und Afrika inzwischen sehr weit verbreitet, obwohl viele dieser Länder keineswegs Demokratien sind und nur schwach ausgeprägte Zivilgesellschaften haben, die eine stärkere ,Demokratisierung' der Regionalorganisation gefordert haben könnten (s. etwa verschiedene Kapitel in Ribeiro Hoffmann u. van der Vleuten 2007). Die Frage ist dann, wie solche Institutionen diesen Status erreichen, welche Rolle bestimmte Vorbilder wie etwa die EU dabei spielen und wie solche Prozesse der Diffusion von bestimmten Designs im Einzelnen ablaufen. 


\subsection{Was bestimmt die Effizienz und Performanz von Regionalorganisationen?}

Während die RDII-Literatur schlicht davon ausgeht, dass Institutionen effizient sind (andernfalls gäbe es sie nicht), betonen andere Autoren staatliche Kapazitäten oder konstatieren fehlende Voraussetzungen für erfolgreiche Regionalintegration, wie etwa die Komplementarität von nationalen Volkswirtschaften (etwa Mattli 1999). Regionalorganisationen außerhalb Europas weisen häufig Effizienzund Performanzprobleme auf. Die ASEAN steht nicht nur im Ruf, eine der erfolgreichsten Regionalorganisationen außerhalb Europas zu sein, sie wird von ihren Kritikern auch gerne als "Quasselbude" bezeichnet, die viel vereinbart, aber nichts umsetzt (Stahl 2010). Einen ähnlich zweifelhaften Ruf hat der Mercosur (Malamud 2005). Der vielleicht extremste Fall in dieser Hinsicht ist jedoch Afrika, wo praktisch alle Länder Mitglied in mehreren, überlappenden Regionalorganisationen sind, deren Ziele teilweise unvereinbar sind, etwa weil sie die Schaffung einer Zollunion anstreben (Bach 2005). Insofern bleibt es aus dieser Perspektive ein Rätsel, warum viele Staaten immer wieder den Weg der regionalen Integration gehen, wenn sie wissen, dass die wirtschaftlichen Voraussetzungen für Integration nicht gegeben sind.

Die Verbindung der weiter oben diskutierten Kausalmechanismen für Diffusion könnte hier eine plausible Erklärung liefern. So kann vermutet werden, dass unterschiedliche Diffusionsmechanismen wie Lernen oder Mimikry ganz unterschiedliche Effekte auf die Effizienz von Institutionen und Politiken haben (Elkins u. Simmons 2005). Die soziologische Literatur theoretisiert dies unter dem Konzept der Entkopplung (Meyer u. Rowan 1977). Wenn für Staatenvertreter die Zugehörigkeit zu einer Gemeinschaft moderner Staaten wichtig ist und sie deshalb die Schaffung von Regionalorganisationen nachahmen, dann ist zu erwarten, dass sie nicht die notwendigen Folgemaßnahmen ergreifen, um die übernommenen Institutionen und Politiken auch zu implementieren. Institutionen werden nicht aus Effektivitätserwägungen geschaffen, sondern um Identität Ausdruck zu verleihen. Dieser theoretische Zugriff hat konkrete Implikationen für die empirische Beobachtung. Beispielsweise ist zu erwarten, dass der Zeitraum zwischen einer institutionellen Neuerung und ihrer Übernahme durch eine andere Regionalorganisation ziemlich kurz ist; die Kürze lässt keine gründliche Evaluierung der Passgenauigkeit dieser Institution zu und beeinflusst damit die Effektivität der übernommenen Institution. Übernahmen, die über den Mechanismus des Lernens erfolgen, zeichnen sich dadurch aus, dass eine institutionelle Neuerung vor der Übernahme genauer auf Passfähigkeit geprüft wird und eventuell Anpassungen erfolgen. In diesem Fall würden wir größere Effizienz erwarten. Allerdings sollte man sich vor tautologischen Schlüssen hüten: Nicht alle Performanzprobleme sind auf Mimikry zurückzuführen und im Einzelnen müssen die Ursachen für Entkopplung genauer untersucht werden.

Auf den Einfluss von Informationen über den relativen Erfolg von Regionalorganisationen sind wir bereits im Zusammenhang mit der Medienberichterstattung eingegangen. Interessant wäre auch zu erfahren, was Regionalorganisationen machen, wenn ihr Modell an Relevanz verliert, wie im Fall der EFTA nach dem Beitritt Großbritanniens zur EG. 


\section{Fazit}

In diesem Artikel haben wir argumentiert, dass die Forschung zum Vergleichenden Regionalismus Ähnlichkeiten zwischen Regionalorganisationen bisher nicht systematisch erfasst und theoretisiert. Bestehende Ansätze analysieren verschiedene Fälle von Regionalismus nach wie vor hauptsächlich als voneinander unabhängige Phänomene, deren Entstehung und Design entweder aus regionalen Dynamiken heraus oder extern durch mächtige Staaten bestimmt sind. Vor diesem Hintergrund haben wir für eine Diffusionsperspektive plädiert und gezeigt, wie diese Perspektive bestehende Fragen neu beantworten und systematisch erweitern kann.

Was kann eine solche Diffusionsperspektive zum Forschungsprogramm der Vergleichenden Regionalismusforschung beitragen? Drei Punkte. Erstens diversifiziert sie die Bandbreite an Analyseperspektiven und ermöglicht es uns dadurch, ein vollständigeres Bild der verschiedenen Faktoren zu erhalten, die globale Regionalismusprozesse beeinflussen. Wir sind nach wie vor weit entfernt von einer "settled comparative theory of regionalism“ (Higgott 2007, S. 75), die diese Faktoren in einer wirklich vergleichenden Perspektive analysiert. Der hier vorgeschlagene Schritt ist explizit auf systematischen Vergleich angelegt und könnte uns etwa helfen, die jeweilige Größe dieser Faktoren neu abzuschätzen.

Zweitens könnte die Diffusionsperspektive dazu beitragen, die Kluft zwischen theoretischen Ansätzen und Disziplinen zu überwinden. EU-Studien und die Vergleichende Regionalismusforschung haben sich bisher seltsamerweise weitgehend ignoriert bzw. es dominieren mögliche Transferanalysen nach dem Motto: „Was können andere Regionalorganisationen von der EU lernen? “ Mit fortschreitender europäischer Integration wird die Relevanz der europäischen Integrationserfahrung für Regionalismus in anderen Teilen der Welt aber zunehmend angezweifelt (s. Warleigh 2004). Die Erfahrung der EU ist relevant, doch sollte sie nicht nur präskriptiv bearbeitet werden, sondern auch empirisch im Sinne von: Welche Institutionen und Politiken der EU werden wann und wie übernommen? Um dies zu tun, brauchen wir das Wissen von Experten der EU und der area studies. Diese neue Forschungsagenda eröffnet vielfältige Möglichkeiten, um die Kluft zwischen theoretischen Ansätzen zu überkommen.

Drittens ermöglicht es die Diffusionsperspektive, bisher vernachlässigte Fragen zur Entstehung von Regionalinstitutionen und ihrem Design, statt ihrer Funktionsweise und ihren Effekten, zu beantworten. In den vergangenen Jahren ist die Institutionenanalyse im Allgemeinen verstärkt dazu übergegangen, Fragen nach dem Design und der Entwicklung von Institutionen bevorzugt zu behandeln (s. Pierson 2004), aber diese Trends haben sich in der Vergleichenden Regionalismusforschung bisher kaum niedergeschlagen (aber s. Acharya u. Johnston 2007). Die Diffusionsperspektive verheißt einen nützlichen Analysewinkel, von dem aus diese Fragen bearbeitet werden können. 


\section{Literatur}

Abbott, Frederick. 1992. Integration Without Institutions: The NAFTA Mutation of the EC Model and the Future of the GATT Regime. American Journal of Comparative Law 40:917-948.

Acharya, Amitav. 2009. Whose Ideas Matter? Agency and Power in Asian Regionalism. Ithaca: Cornell University Press.

Acharya, Amitav, und Alastair Iain Johnston (Hrsg.). 2007. Crafting Cooperation: The Design and Effect of Regional Institutions in Comparative Perspective. Cambridge: Cambridge University Press.

Aldrich, Howard. 1979. Organizations and Environments. Englewood Cliffs: Prentice Hall.

Alecu de Flers, Nicole, und Elfriede Regelsberger. 2005. The EU and Inter-Regional Cooperation. In International Relations and the European Union, Hrsg. Christopher Hill und Michael Smith, 317-324. Oxford: Oxford University Press.

Alter, Karen. 2008. Jurist Advocacy Movements in Europe and the Andes: How Lawyers Help Promote International Legal Integration. Center on Law and Globalization Research Paper 08-05. Champaign/Chicago: Center on Law and Globalization.

Alter, Karen, und Laurence Helfer. 2010. Nature or Nurture? Judicial Lawmaking in the European Court of Justice and the Andean Tribunal of Justice. International Organization 64:563-592.

Avery, William. 1973. Extra-Regional Transfer of Integrative Behavior. International Organization 27:549-556.

Bach, Daniel. 2005. The Global Politics of Regionalism: Africa. In Global Politics of Regionalism: Theory and Practice. Hrsg. Mary Farrell, Björn Hettne und Luk van Langenhove, 171-186. London: Pluto.

Baldwin, Richard. 1993. A Domino Theory of Regionalism. CEPR Discussion Paper 857. London: Centre for Economic Policy Research.

Beeson, Mark. 2003. ASEAN Plus Three and the Rise of Reactionary Regionalism. Contemporary Southeast Asia 25:251-268.

Bhagwati, Jagdish. 1994. Regionalism and Multilateralism: An Overview. In Asia Pacific Regionalism: Readings in International Economic Relations, Hrsg. Ross Garnaut und Peter Drysdale, 145-166. Canberra: Australia-Japan Research Centre.

Bicchi, Federica. 2006. 'Our Size Fits All': Normative Power Europe and the Mediterranean. Journal of European Public Policy 13:286-303.

Börzel, Tanja, und Thomas Risse. 2009. Diffusing (Inter-) Regionalism. KFG Working Paper Series 7. Berlin: Freie Universität Berlin, Kolleg-Forschergruppe „The Transformative Power of Europe“.

Börzel, Tanja, und Thomas Risse. 2011. From Europeanization to Diffusion: Introduction. West European Politics (im Erscheinen).

Botto, Mercedes. 2009. The Role of Epistemic Communities in the 'Makability' of Mercosur. In The EU and World Regionalism: The Makability of Regions in the 21st Century, Hrsg. Philippe de Lombaerde und Michael Schulz, 171-186. Aldershot: Ashgate.

Bowles, Paul. 2002. Asia's Post-Crisis Regionalism: Bringing the State Back in, Keeping the (United) States Out. Review of International Political Economy 9:244-270.

Busse, Nikolas. 1999. Constructivism and Southeast Asian Security. The Pacific Review 12:39-60. 
Büthe, Tim, und Helen Milner. 2008. The Politics of Foreign Direct Investment into Developing Countries: Increasing FDI through International Trade Agreements? American Journal of Political Science 52:741-762.

Checkel, Jeffrey. 2005. International Institutions and Socialization in Europe: Introduction and Framework. International Organization 59:801-826.

Coleman, William, und Geoffrey Underhill (Hrsg.). 1998. Regionalism and Global Economic Integration: Europe, Asia and the Americas. London: Routledge.

Collier, David, und Richard E. Messick. 1975. Prerequisites Versus Diffusion: Testing Alternative Explanations of Social Security Adoption. American Political Science Review 69:1299-1315.

Dash, Kishore. 1996. The Political Economy of Regional Cooperation in South Asia. Pacific Affairs 69:185-209.

Dent, Christopher. 2008. The Asian Development Bank and Developmental Regionalism in East Asia. Third World Quarterly 29:767-786.

Dimon, Denise. 2006. EU and US Regionalism: The Case of Latin America. The International Trade Journal 20:185-218.

Doidge, Mathew. 2007. Joined at the Hip: Regionalism and Interregionalism. Journal of European Integration 29:229-248.

Dosch, Jörn. 1997. Die Asean: Bilanz eines Erfolges. Akteure, Interessenlagen, Kooperationsbeziehungen. Hamburg: Abera Verlag.

Dri, Clarissa. 2009. Limits of the Institutional Mimesis of the European Union: The Case of the Mercosur Parliament. Latin American Policy 1:52-74.

Duina, Francesco. 2006. The Social Construction of Free Trade. The European Union, Nafta and Mercosur. Princeton: Princeton University Press.

Edwards, Geoffrey, and Elfriede Regelsberger (Hrsg.). 1990. Europe's Global Links: The European Community and Inter-Regional Cooperation. London: Pinter.

Elkins, Zachary, und Beth Simmons. 2005. On Waves, Clusters, and Diffusion: A Conceptual Framework. Annals of the American Academy of Political and Social Science 598:33-51.

Farrell, Mary. 2007. From EU Model to External Policy? Promoting Regional Integration in the Rest of the World. In The State of the European Union, Hrsg. Sophie Meunier und Kathleen McNamara, 299-316. Oxford: Oxford University Press.

Fawcett, Louise 1995. Regionalism in Historical Perspective. In Regionalism in World Politics: Regional Organization and International Order, Hrsg. Louise Fawcett und Andrew Hurrell, 9-36. Oxford: Oxford University Press.

Finnemore, Martha. 1993. International Organizations as Teachers of Norms: The United Nations Educational, Scientific, and Cultural Organisation and Science Policy. International Organization 47:565-597.

Franzese, Robert, und Jude Hays. 2008. Empirical Models of Spatial Interdependence. In Oxford Handbook of Political Methodology, Hrsg. Janet Box-Steffensmeier, Henry E. Brady und David Collier, 570-604. Oxford: Oxford University Press.

Gilson, Julie. 2005. New Interregionalism? The EU and East Asia. Journal of European Integration 27:307-326.

Goetze, Stefan, und Berthold Rittberger. 2010. A Matter of Habit? The Sociological Foundations of Empowering the European Parliament. Comparative European Politics $8: 37-54$ 
Goldstein, Judith, und Robert Keohane. 1993. Ideas and Foreign Policy: An Analytical Framework. In Ideas and Foreign Policy: Beliefs, Institutions and Political Change, Hrsg. Judith Goldstein und Robert Keohane, 3-30. Ithaca: Cornell University Press.

Grieco, Joseph. 1997. Systemic Sources of Variation in Regional Institutionalization in Western Europe, East Asia, and the Americas. In The Political Economy of Regionalism, Hrsg. Edward Mansfield und Helen Milner, 164-87. New York: Columbia University Press.

Grugel, Jean. 2004. New Regionalism and Modes of Governance: Comparing US and EU Strategies in Latin America. European Journal of International Relations 10:603-626.

Haas, Ernst. 1961. International Integration: The European and the Universal Process. International Organization 15:366-392.

Haas, Peter. 1992. Introduction: Epistemic Communities and International Policy Coordination. International Organization 46:1-35.

Hall, Peter. 1993. Policy Paradigms, Social Learning, and the State: The Case of Economic Policymaking in Britain. Comparative Politics 25:275-296.

Hänggi, Heiner. 2007. Regionalism through Interregionalism: East Asia and ASEM. In Regionalism in East Asia: Paradigm Shifting?, Hrsg. Liu Fu-Kuo und Philippe Régnier, 197-219. London: Routledge.

Hänggi, Heiner, Ralf Roloff, und Jürgen Rüland (Hrsg.). 2006. Interregionalism and International Relations. London: Routledge.

Hettne, Björn. 1999. Globalization and the New Regionalism: The Second Great Transformation. In Globalism and the New Regionalism, Hrsg. Björn Hettne, Andras Sapir und Osvaldo Sunkel. New York: St. Martin's Press.

Higgott, Richard. 2007. Alternative Models of Regional Cooperation? The Limits of Regional Institutionalization in East Asia. In European Union and New Regionalism: Regional Actors and Global Governance in a Post-Hegemonic Era, Hrsg. Mario Telò, 75-106. Aldershot: Ashgate.

Hoffmann, Stanley. 1966. Obstinate or Obsolete? The Fate of the Nation State and the Case of Western Europe. Daedalus 95:862-915.

Holzinger, Katharina, Helge Jörgens, und Christoph Knill (Hrsg.). 2007. Transfer, Diffusion und Konvergenz von Politiken. Politische Vierteljahresschrift Sonderheft 38. Wiesbaden: VS Verlag für Sozialwissenschaften.

Huntington, Samuel P. 1991. The Third Wave: Democratization in the Late Twentieth Century. Norman: University of Oklahoma Press.

Jahn, Detlef. 2009. Globalisierung als Galton-Problem: Regionale und Temporale Diffusionsschübe. In Methoden der Vergleichenden Politik- und Sozialwissenschaft, Hrsg. Susanne Pickel, Gert Pickel, Hans-Joachim Lauth und Detlef Jahn, 87-112. Wiesbaden: VS Verlag für Sozialwissenschaften.

Jetschke, Anja. 2009. Institutionalizing ASEAN: Celebrating Europe through Network Governance. Cambridge Review of International Affairs 22:407-26.

Jetschke, Anja. 2010. Do Regional Organizations Travel? European Integration, Diffusion and Regional Organization in Asia. KFG Working Paper Series 17. Berlin: Freie Universität Berlin, Kolleg-Forschergruppe „The Transformative Power of Europe“.

Jetschke, Anja, und Philomena Murray. 2011. Diffusing Regional Integration: The EU and Southeast Asia. West European Politics 34 (im Erscheinen).

Job, Brian. 2003. Track 2 Diplomacy: Ideational Contribution to the Evolving Asia Security Order. In Asian Security Order: Instrumental and Normative Features, Hrsg. Muthia Alagappa, 241-79. Stanford: Stanford University Press. 
Jolliff, Brandy, und Joseph Jupille. 2010. Method, Model and Mentor: The EU as Promoter of Regional Integration. Beitrag präsentiert auf der Internationalen Konferenz „Diffusion of Regional Integration“, 8.-9.12.2010, Berlin.

Kahler, Miles. 2000. Legalization as Strategy: The Asia-Pacific Case. International Organization 54:549-571.

Kaiser, Karl. 1968. The Interaction of Regional Subsystems: Some Preliminary Notes on Recurrent Patterns and the Role of the Superpowers. World Politics 21:84-107.

Katsumata, Hiro. 2010. Mimetic Adoption and Norm Diffusion: 'Western' Security Cooperation in Southeast Asia? Review of International Studies 37:557-576.

Katz, Elihu, Martin Levin, und Herbert Hamilton. 1963. Traditions of Research on the Diffusion of Innovation. American Sociological Review 28:237-252.

Katzenstein, Peter. 2005. A World of Regions. Asia and Europe in the American Imperium. Ithaca: Cornell University Press.

Keohane, Robert. 1984. After Hegemony: Cooperation and Discord in the World Political Economy. Princeton: Princeton University Press.

Khoman, Thanat. 1992. ASEAN: Conception and Evolution. In The ASEAN Reader, Hrsg. Kernial S. Sandhu, Sharon Siddique, Chandran Jeshurun, Ananda Rajah, Joseph Tan und Pushpa Thambipillai, xvii-xxii. Singapur: Institute for Southeast Asian Studies.

Koremenos, Barbara, Charles Lipson, und Duncan Snidal. 2001. The Rational Design of International Institutions. International Organization 55:761-799.

Krapohl, Sebastian. 2008. New Institutionalism Meets International Political Economy: A New Approach and Research Agenda for the Study of Regional Integration In-and Outside Europe. Beitrag präsentiert auf der GARNET Conference "The European Union in International Affairs", 22-24.4.2010, Brüssel.

Kupchan, Charles. 1997. Regionalizing Europe's Security: The Case for a New Mitteleuropa. In The Political Economy of Regionalism, Hrsg. Edward Mansfield und Helen Milner, 209-238. New York: Columbia University Press.

Lawrence, Robert. 1996. Regionalism, Multilateralism, and Deeper Integration. Washington, DC: Brookings Institution.

Lenz, Tobias. 2011. Spurred Emulation: The EU and Regional Integration in Mercosur and SADC. West European Politics 34 (im Erscheinen).

Lombaerde, Philippe de, und Michael Schulz (Hrsg.). 2009. The EU and World Regionalism: The Makability of Regions in the 21st Century. Aldershot: Ashgate.

Lucarelli, Sonia. 2007. The European Union in the Eyes of Others: Towards Filling a Gap in the Literature. European Foreign Affairs Review 12:249-270.

Malamud, Andres. 2005. Mercosur Turns 15: Between Rising Rhetoric and Declining Achievement. Cambridge Review of International Affairs 18:421-436.

Mansfield, Edward, und Helen Milner. 1999. The New Wave of Regionalism. International Organization 53:589-627.

Mansfield, Edward, Helen Milner, und Jon Pevehouse. 2008. Democracy, Veto Players and the Depth of Regional Integration. The World Economy 31:67-96.

Marschall, Stefan. 2005. Transnationale Repräsentation in Parlamentarischen Versammlungen. Baden-Baden: Nomos.

Mattli, Walter. 1999. The Logic of Regional Integration: Europe and Beyond. Cambridge: Cambridge University Press.

Meseguer, Covadonga. 2009. Learning, Policy Making, and Market Reforms. Cambridge: Cambridge University Press. 
Meyer, John W., und Brian Rowan. 1977. Institutionalized Organizations: Formal Structure as Myth and Ceremony. American Journal of Sociology 83:340-363.

Mols, Manfred. 1996. Integration und Kooperation in zwei Kontinenten: Das Streben nach Einheit in Lateinamerika und in Südostasien. Stuttgart: Steiner.

Moravcsik, Andrew. 1998. The Choice for Europe: Social Purpose and State Power from Messina to Maastricht. Ithaca: Cornell University Press.

Most, Benjamin, und Harvey Starr. 1990. Theoretical and Logical Issues in the Study of International Diffusion. Journal of Theoretical Politics 2:391-412.

Nabers, Dirk, und Andreas Ufen (Hrsg.). 2005. Regionale Integration: Neue Dynamiken in Afrika, Asien und Lateinamerika. Hamburg: Deutsches Übersee-Institut.

Nye, Joseph. 1965. Patterns and Catalysts in Regional Integration. International Organization 19:870-884.

O’Loughlin, John, Michael D. Ward, Corey L. Lofdahl, Jordin S. Cohen, David S. Brown David Reilly, Kristian S. Gleditsch, und Michael Shin. 1998. The Diffusion of Democracy, 1946-1994. Annals of the Association of American Geographers, 88:545-574.

Packer, Corinne, und Donald Rukare. 2002. The New African Union and Its Constitutive Act. The American Journal of International Law 96:365-379.

Pierson, Paul. 2004. Politics in Time: History, Institutions, and Social Analysis. Princeton: Princeton University Press.

Pinder, John. 1968. EEC and Comecon. In International Regionalism: Readings, Hrsg. Joseph Nye, 22-42. Boston: Little, Brown \& Company.

Ribeiro Hoffmann, Andrea, und Anna van der Vleuten (Hrsg.). 2007. Closing or Widening the Gap? Legitimacy and Democracy in Regional Integration Organizations. Aldershot: Ashgate.

Rogers, Everett. 1983. Diffusion of Innovations. New York: Free Press.

Rüland, Jürgen. 2010. Balancers, Multilateral Utilities or Regional Identity Builders? International Relations and the Study of Interregionalism. Journal of European Public Policy 17:1271-1283.

Saldías, Osvaldo. 2010. Networks, Courts and Regional Integration: Explaining the Establishment of the Andean Court of Justice. KFG Working Paper Series 20. Berlin: Freie Universität Berlin, Kolleg-Forschergruppe „The Transformative Power of Europe“.

Sampson, Gary, und Stephen Woolcock (Hrsg.). 2003. Regionalism, Multilateralism and Economic Integration: The Recent Experience. Tokyo: United Nations University Press.

Sanchez-Bajo, Claudio. 1999. The European Union and Mercosur: A Case of Inter-Regionalism. Third World Quarterly 20:927-941.

Sandholtz, Wayne, und Alec Stone Sweet (Hrsg.). 1998. European Integration and Supranational Governance. Oxford: Oxford University Press.

Sandholtz, Wayne, und John Zysman. 1989. 1992: Recasting the European Bargain. World Politics 42:95-128.

Sbragia, Alberta. 2002. "Competitive Regionalism, Trade Liberalization, and Globalization: The EU and the Americas," delivered at the Conference of Europeanists March 11-13, 2004, Chicago.

Sbragia, Alberta. 2008. Review Article: Comparative Regionalism: What Might It Be? Journal of Common Market Studies 46:29-49.

Schimmelfennig, Frank, und Ulrich Sedelmeier. 2005. Introduction: Conceptualizing the Europeanization of Central and Eastern Europe. In The Europeanization of Central and Eastern Europe, Hrsg. Frank Schimmelfennig und Ulrich Sedelmeier, 1-28. Ithaca: Cornell University Press. 
Schirm, Stefan. 2002. Globalization and the New Regionalism: Global Markets, Domestic Politics and Regional Cooperation. Cambridge: Polity Press.

Simmons, Beth, Frank Dobbin, und Geoffrey Garrett. 2006. Introduction: The International Diffusion of Liberalism. International Organization 60:781-810.

Smith, Karen E. 2008. European Union Foreign Policy in a Changing World. Cambridge: Polity Press.

Söderbaum, Fredrik, und Luk van Langenhove (Hrsg.). 2006. The EU as a Global Player: The Politics of Interregionalism. London: Routledge.

Solingen, Etel. 2008. The Genesis, Design and Effects of Regional Institutions: Lessons from East Asia and the Middle East. International Studies Quarterly 52:261-294.

Sommerer, Thomas. 2011. Können Staaten voneinander lernen? Eine Vergleichende Analyse der Umweltpolitik in 24 Ländern. Wiesbaden: VS Verlag für Sozialwissenschaften.

Stahl, Bernhard. 2010. Die Gemeinschaft Südostasiatischer Staaten (ASEAN): Erfolg und Probleme einer überforderten Institution. In Multilaterale Institutionen in OstasienPazifik, Hrsg. Dirk Nabers, 17-54. Wiesbaden: VS Verlag für Sozialwissenschaften.

Strang, David, und John D. Meyer. 1993. Institutional Conditions for Diffusion. Theory and Society 22:487-511.

Telò, Mario (Hrsg.). 2001. European Union and New Regionalism: Regional Actors and Global Governance in a Post-Hegemonic Era. Aldershot: Ashgate.

Trivedi, Sonu. 2006. SAARC-COMESA. Journal of Developing Societies 22:57-73.

Walker, Jack L. 1969. The Diffusion of Innovations among the American States. The American Political Science Review 63:880-899.

Warleigh, Alex. 2004. In Defence of Intra-Disciplinarity: 'European Studies', the 'New Regionalism' and the Issue of Democratisation. Cambridge Review of International Affairs 17:301-318.

Weyland, Kurt. 2008. Toward a New Theory of Institutional Change. World Politics 60:281-314.

Yeo, Lay Hwee. 2008. EU-ASEAN Relations and Policy-Learning. In Europe-Asia Relations: Building Multilateralism, Hrsg. Richard Balme und Brian Bridges, 83-102. Basingstoke: Palgrave Macmillan.

\section{Autorenangaben:}

Dr. Anja Jetschke,

GIGA Leibniz Institut für Globale und Regionale Studien, Neuer Jungfernstieg 21,

20354 Hamburg, jetschke@giga-hamburg.de

[Korrespondenzautorin]

Tobias Lenz, M. Phil., University of Oxford, Department of Politics and International Relations, St. Antony's College, Oxford, OX2 6JF, England, tobias.lenz@sant.ox.ac.uk 